Research paper

\title{
Evidence of distinct profiles of Posttraumatic Stress Disorder (PTSD) and Complex Posttraumatic Stress Disorder (CPTSD) based on the new ICD-11 Trauma Questionnaire (ICD-TQ)
}

\author{
a Edinburgh Napier University, School of Health \& Social Care,Edinburgh, UK \\ b NHS Lothian, Rivers Centre for Traumatic Stress, Edinburgh, UK \\ c Ulster University, School of Psychology, Derry, UK \\ d National College of Ireland, School of Business, Dublin, Ireland \\ e Psychology and Counselling Directorate, Cardiff and Vale University Health Board, Cardiff, UK \\ ${ }^{\mathrm{f}}$ Cardiff University, School of Medicine, Cardiff, UK \\ g University College London, Clinical, Education \& Health Psychology, London, UK \\ h New York University, School of Medicine, USA

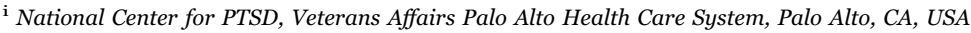

Thanos Karatzias $^{\mathrm{a}, \mathrm{b}, *}$, Mark Shevlin ${ }^{\mathrm{c}}$, Claire Fyvie ${ }^{\mathrm{b}}$, Philip Hyland ${ }^{\mathrm{d}}$, Erifili Efthymiadou ${ }^{\mathrm{b}}$, Danielle Wilson $^{\mathrm{b}}$, Neil Roberts ${ }^{\mathrm{e}}$, Jonathan I. Bisson ${ }^{\mathrm{f}}$, Chris R. Brewin ${ }^{\mathrm{g}}$, Marylene Cloitre ${ }^{\mathrm{h}, \mathrm{i}}$

\section{A R T I C L E I N F O}

Keywords:

ICD-11

Posttraumatic Stress Disorder (PTSD) and

Complex Posttraumatic Stress Disorder (CPTSD)

ICD-11 Trauma Questionnaire (ICD-TQ)

\begin{abstract}
A B S T R A C T
Background: The WHO International Classification of Diseases, 11th version (ICD-11), has proposed two related diagnoses following exposure to traumatic events; Posttraumatic Stress Disorder (PTSD) and Complex PTSD (CPTSD). We set out to explore whether the newly developed ICD-11 Trauma Questionnaire (ICD-TQ) can distinguish between classes of individuals according to the PTSD and CPTSD symptom profiles as per ICD11 proposals based on latent class analysis. We also hypothesized that the CPTSD class would report more frequent and a greater number of different types of childhood trauma as well as higher levels of functional impairment. Methods Participants in this study were a sample of individuals who were referred for psychological therapy to a National Health Service (NHS) trauma centre in Scotland (N=193). Participants completed the ICD-TQ as well as measures of life events and functioning.

Results: Overall, results indicate that using the newly developed ICD-TQ, two subgroups of treatment-seeking individuals could be empirically distinguished based on different patterns of symptom endorsement; a small group high in PTSD symptoms only and a larger group high in CPTSD symptoms. In addition, CPTSD was more strongly associated with more frequent and a greater accumulation of different types of childhood traumatic experiences and poorer functional impairment.

Limitations: Sample predominantly consisted of people who had experienced childhood psychological trauma or been multiply traumatised in childhood and adulthood.

Conclusions: CPTSD is highly prevalent in treatment seeking populations who have been multiply traumatised in childhood and adulthood and appropriate interventions should now be developed to aid recovery from this debilitating condition.
\end{abstract}

\section{Introduction}

Two 'sibling disorders' have been proposed for ICD-11; Posttraumatic Stress Disorder (PTSD) and Complex PTSD (CPTSD) (Maercker et al., 2013). The organizing principles for the ICD-11 revisions were that diagnoses should be consistent with clinicians' mental health taxonomies, limited in the number of symptoms included, and based on distinctions important for management and treatment (Reed, 2010). The ICD-11 model of PTSD includes symptoms reflecting three clusters: (1) re-experiencing of the trauma in the present (Re), (2) avoidance of traumatic reminders (Av), and (3) a persistent sense of threat that is manifested by increased arousal and hypervigilance (Th). These symptoms define PTSD as a response characterised by some degree of fear or horror related to a specific

\footnotetext{
* Correspondence to: Edinburgh Napier University, Sighthill Campus, Sighthill Court, Edinburgh EH11 4BN, Scotland, UK.

E-mail address: Email.t.karatzias@napier.ac.uk (T. Karatzias).
} 
traumatic event. In contrast, the symptom profile of CPTSD includes the core PTSD symptoms plus three additional symptoms that identify 'disturbances in self-organization' (DSO): (1) affective dysregulation (AD), (2) negative self-concept (NSC), and (3) disturbances in relationships (DR).

The DSO component of the ICD-11 model is consistent with the plethora of research findings that indicate how prolonged interpersonal trauma, particularly of an early relational type, can result in the development and maintenance of negative and denigrating view of self, and fearful and threating interpretation of others. Childhood sexual abuse has been shown to be associated with shame (Andrews, 1998), guilt (Street, Gibson, and Holohan, 2005), adoption of defensive submissive strategies (Gilbert, 2000), perceptions of low self-worth (Kucharska, 2015), self-directed disgust (Badour et al., 2014) and fearful attitudes toward relationships (Harris and Valentiner, 2002). Furthermore, the role of interpersonal trauma in emotional dysregulation is well established (Dvir et al., 2014). Therefore, the concept of DSO can be seen as a convenient summary of the multitude of deleterious effects of prolonged interpersonal trauma.

More formally the distinction between PTSD and Complex PTSD was first articulated by Herman (1992) who proposed that prolonged interpersonal traumatic stressors (e.g., childhood abuse, domestic violence, being a prisoner of war) negatively impacted self-organization, independent of PTSD symptoms. Data from the DSM-IV field trials indicated that those with chronic trauma exposure reported high rates of symptoms representative of disturbances in affective, self and relational domains compared to those with other types of trauma histories (Roth et al., 1997). Since that time, data has been accumulating indicating the presence of salient disturbances in these domains as particularly associated with childhood trauma (e.g., Briere and Rickards, 2007; Cloitre et al., 1997; Kaltman et al., 2005) and some data indicating disturbances in these domains in samples defined by adult-onset sustained interpersonal violence such as civilians exposed to war (e.g., Morina and Ford, 2008).

Given the potential for ICD formulations to become the primary diagnostic classification system used in the field of psychotraumatology (Wolf et al., 2015), a thorough empirical assessment of the ICD-11 models of trauma-based disorders is required. The ICD-11 model of CPTSD predicts that there should be evidence of qualitatively different patterns, or profiles, of symptom endorsement and these different profiles should be related to the nature of the trauma exposure. Such evidence is usually provided by the results from mixture models that identify different homogeneous sub-populations that share similar patterns of symptom endorsement. Specifically, it is predicted that there would be evidence of PTSD characterised by high endorsement of PTSD symptoms and low endorsement of DSO symptoms. CPTSD would be characterised by high endorsement of both PTSD and DSO symptoms. Also, the CPTSD profile of symptom endorsement should be more strongly associated with sustained, repeated, and multiple forms of traumatic exposures. It is also possible to find other profiles, such as low endorsement of all symptoms, without invalidating the CPTSD model.

There have been several studies that have tested the ICD-11 model of CPTSD using mixture models, most commonly using latent class analysis (LCA) and latent profile analysis (LPA). These studies and their findings are summarised in Table 1 .

The six studies that employed mixture models, utilizing seven trauma samples found support for the distinction between ICD-11 PTSD and CPTSD while one study has called this into question (Wolf et al., 2015). Overall, the research evidence for the ICD-11 model of CPTSD is largely supportive as the findings from the mixture models support the qualitative distinction between PTSD and CPTSD.

The present study aimed to determine if there are qualitatively different groups of participants, or classes, with symptom endorsement that reflect PTSD and CPTSD using the only self-report scale (i.e. ICD11 Trauma Questionnaire (ICD-TQ); Cloitre, Roberts, Bisson, and
Brewin, 2014) that has been developed to measure CPTSD as proposed by the ICD-11. Analyses were based on data from a sample of outpatients seeking psychological treatment for distress following traumatic events. It was predicted that (1) separate classes representing PTSD (high probabilities of meeting diagnostic criteria for the three PTSD symptom clusters and low probabilities of meeting diagnostic criteria for the three DSO symptom clusters) and CPTSD (high probabilities of meeting diagnostic criteria for the three PTSD and three DSO symptom clusters) would be found, (2) the CPTSD class would report higher rates of childhood trauma (individual and cumulative) and stressful life events, and (3) the CPTSD class would report higher levels of functional impairment (home management, social leisure activities, private leisure activities and relationships with others). The study also aimed to examine differences between the PTSD and CPTSD classes on a range of socio-demographic variables.

\section{Method}

\subsection{Participants and procedure}

Participants in this study were individuals who were referred by general practitioners, psychiatrists or psychologists for psychological therapy to a National Health Service (NHS) trauma centre in Scotland. All 230 new patients over the 18 month recruitment period were sent a letter and invited to complete a set of standardised measures. Twentytwo did not respond and 13 provided unusable data due to large amounts of missing responses, and 2 had missing scores on the ICD TQ which resulted in a final sample size of 193.

The mean age of the sample was 41 years $(\mathrm{SD}=12.4)$ and there were more females (65.1\%) than males. Most of the sample were born in the United Kingdom (88.7\%) and of these most were from Scotland (79\%). The highest level of academic attainment was varied: school (38.5\%), College (30.2\%), and University (30.2\%). Approximately one third of the sample was in employment (full-time $20.2 \%$, part-time $13 \%$ ), $38.9 \%$ were unemployed, $7.3 \%$ were retired, and $5.7 \%$ were in voluntary work ( $15 \%$ reported 'None of these'). Almost half of the sample were single (48.2\%), $22.3 \%$ were married, $12.4 \%$ were divorced, and $9.8 \%$ were co-habiting. Most participants were either living with partner or with their family (41\%), $34.7 \%$ were living alone (and $24.4 \%$ reported 'Other').

\subsection{Measures}

\subsubsection{ICD 11 Trauma Questionnaire (ICD-TQ; Version 1.2, Cloitre} et al., 2014)

The ICD-TQ is a 23-item self-report measure for the screening of ICD-11 PTSD and CPTSD symptomatology. Six items represent the three clusters of PTSD including Re-experiencing (RE) (items P1-P2), Avoidance (AV) (items P3-P4), and Sense of Threat (Th) that is manifested by increased arousal and hypervigilance (items P5-P6). CPTSD includes PTSD as well as three clusters reflecting DSO. Sixteen items represent the three DSO clusters including affective dysregulation ( $\mathrm{AD}$, items $\mathrm{C} 1-\mathrm{C} 9$ ), negative self-concept (NSC, items C10-C13), and disturbances in relationships (DR, items C14-C16). Symptom endorsement is scored on a Likert scale, indicating how much a symptom has been bothersome in the past month, with scores ranging from 0 (not at all) to 4 (extremely). The scale can be used to estimate a self-report ICD-11 PTSD or CPTSD diagnosis by recoding the Likert scores into six binary variables representing each of the 3 PTSD and DSO symptom clusters based on the following cut-off scores. A diagnosis of PTSD requires a score of $\geq 2$ (moderately (2), quite a lot (3), extremely (4)) for at least one symptom in each of its three clusters. A diagnosis of CPTSD requires PTSD and the following scores for each of the three DSO clusters. As for PTSD component, an item requires a score of $\geq 2$ to be positive. The proposed algorithm for each DSO cluster requires a sum that is half of the total possible score. $\mathrm{AD}$ requires a 


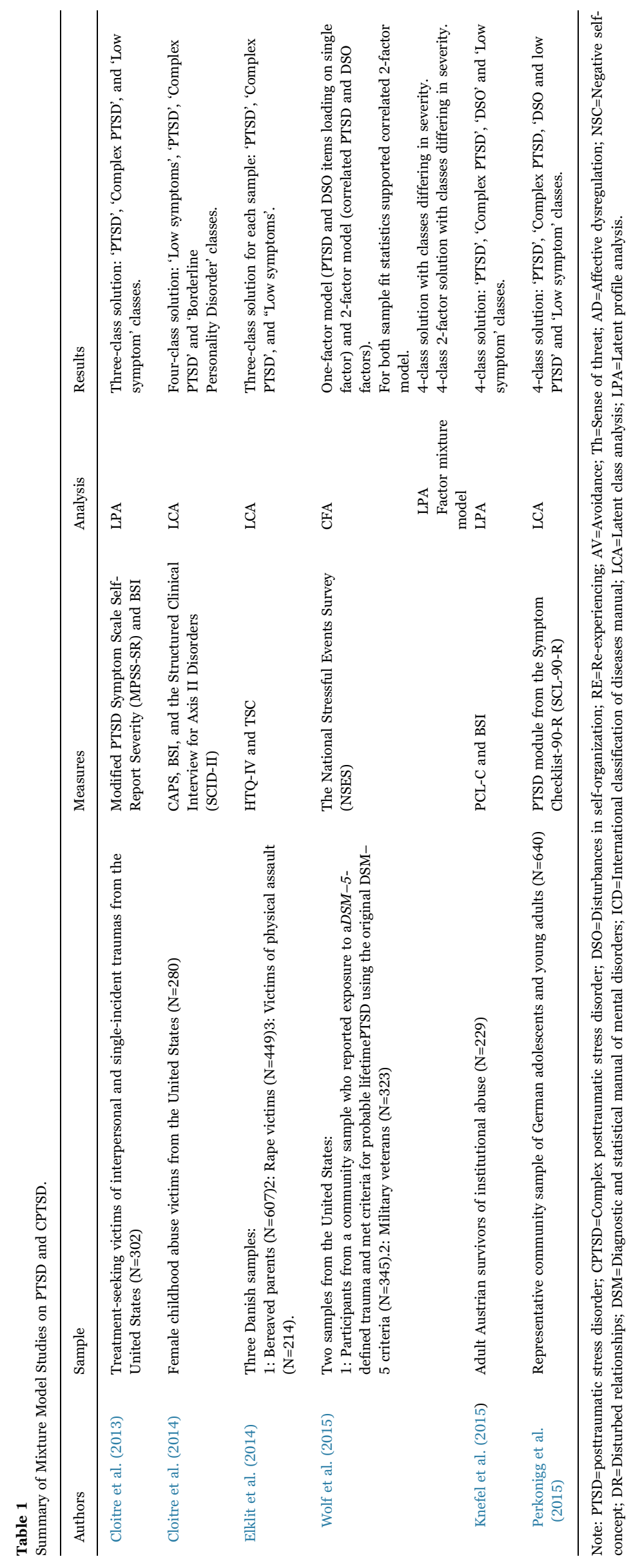


score $\geq 10$ on items $1-5$ (hyper-activation) or a score of $\geq 8$ on items $6-$ 9 (deactivation), for the 4 NSC items a score $\geq 8$ and for the 3 DR items a score $\geq 10$ are required. Cronbach's alpha reliability estimates for the PTSD indicators were modest $(\mathrm{RE}=.55, \mathrm{AV}=.63$, and $\mathrm{Th}=.78)$, but higher for the $\mathrm{DSO}$ indicators $(\mathrm{AD}=.79, \mathrm{NSC}=.91$, and $\mathrm{DR}=.83)$. The estimates of reliability for the PTSD indicators are likely to be underestimates of true reliability due to the small number of variables (Eisinga et al., 2012).

\subsubsection{Childhood Trauma Questionnaire (CTQ: Bernstein and Fink, 1998)}

The CTQ is a 28-item self-report questionnaire that assesses exposure to range of different childhood traumas. The scale produces five subscales, each with five items: Emotional Abuse, Physical Abuse, Sexual Abuse, Emotional Neglect, Physical Neglect. Items are responded to using a 5-point scale ranging from "never true" (1) to "very often true" in regards to the endorsed frequency of the event (5) and the mean scores for each subscale were calculated. The CTQ also has three items that assess minimization/denial. These were not used in the analyses. Cronbach's a reliability of the scales was high in this sample; Emotional Abuse (.90), Physical Abuse (.85), Sexual Abuse (.97), Emotional Neglect (.92), Physical Neglect (.83). There were missing data on 15 items ranging from $3.6 \%$ to $4.1 \%$. To reduce the impact of missing data a conservative approach was taken whereby missing data were assumed to represent non-endorsement of the item.

\subsubsection{The Life Events Checklist (LEC: Gray et al., 2004)}

The LEC is a 17-item self-report measure designed to screen for potentially traumatic events in a respondent's lifetime. The LEC assesses life time exposure to 16 traumatic events (e.g. Natural disaster, Physical assault, Life threatening illness/injury) and the 17th item, "Any other very stressful event/experience", can be used to indicate exposure to a trauma that was not listed. For each item, the respondent checks whether the event 'Happened to me' (1), 'Witnessed it happening to somebody else' (2), 'Learned about it happening to someone close to me' (3), 'Part of my job' (4), 'Not sure it applies' (5), 'Does not apply to my experience' (6). In order to create a summed total to represent the number of different life events that has been experienced the items were recoded into binary variables with 'Happened to me' responses being coded as 1 and all other responses coded as 0 . This produced a single total cumulative index variable with possible scores ranging from 0 to 16 ; item 17 was not included as the nature of the trauma could not be identified. Cronbach's $\alpha$ of the scale was moderate (.68).

\subsubsection{Work and Social Adjustment Scale (WSAS: Mundt et al., 2002)}

The WSAS is 5-item self-report scale that assesses perceived functional impairment in five domains; work, home management, social leisure activities, private leisure activities and relationships with others. Each domain is assessed using a single item and the participant is asked to "...determine on the scale provided how much your problem affects your ability to carry out the activity. The response scale ranges from 'Not at all' (0) to 'Very severely' (8). The WSAS has been found to provide reliable and valid scores and be a useful indicator of global dysfunction (Jansson-Fröjmark, 2014; Zahra et al., 2014). Due to the low employment rates in this sample $33.2 \%$ were in full or part-time employment) the scores on the work domain were not used. Cronbach's $\alpha$ of the remaining 4 items was acceptable (.74).

\subsection{Analysis}

Latent class analysis (LCA) is a statistical method used to identify homogeneous groups, or classes, from multivariate categorical data. The analysis involved two linked elements. First, an LCA was conducted to determine the number of classes based on the six dimensions of the ICD-11 CPTSD Scale. Binary variables were computed based on the cut-offs. The fit of six models (1-class model through to 6-class model) was assessed. The models were estimated using robust maximum likelihood (Yuan and Bentler, 2000). Missing data on the ICDTQ was low (PTSD 1\%, and DSO 1\%) and the models were estimated using all available information. To avoid solutions based on local maxima, 500 random sets of starting values were used initially and 100 final stage optimizations. The relative fit of the models was compared by using three information theory based fit statistics: the Akaike Information Criterion (AIC; Akaike, 1987), the Bayesian Information Criterion (BIC; Schwartz, 1978) and sample size adjusted Bayesian Information Criterion (ssaBIC; Sclove, 1987). The model that produces the lowest values can be judged the best model. Evidence from simulation studies have indicated that the BIC was the best information criterion for identifying the correct number of classes (Nylund et al., 2007). In addition, the Lo-Mendell-Rubin adjusted likelihood ratio test (LMR-A; Lo et al., 2001) was used to compare models with increasing numbers of latent classes. When a non-significant value $(p>.05)$ occurs this suggests that the model with one less class should be accepted. All analyses were conducted using Mplus 7.00 (Muthén and Muthén, 2012). Second, differences between classes were examined on demographic variables (age, gender, employment, educational attainment, living status), psychotropic medication, childhood trauma (CTQ subscales), trauma experiences (scores from the LEC), and work and social adjustment (WSAS scores) using chi-square and $t$-tests.

\section{Results}

The participants reported exposure to multiple traumatic events. The mean number of traumas reported using the Life Events Checklist was $5.40(\mathrm{SD}=2.60)$, with only a small number $(6.2 \%)$ reporting exposure to a single traumatic event; a total of $71.8 \%$ of the sample reported experiencing between 3 and 8 traumatic events. Scores from the CTQ indicate that there were also high levels of childhood trauma, particularly emotional abuse and emotional neglect: Mean (SD): Emotional Abuse 2.77 (1.35), Physical Abuse 2.20 (1.18), Sexual Abuse 2.43 (1.61), Emotional Neglect 2.64 (1.26), and Physical Neglect 1.89 (.99). Endorsement rates for any item (score $>1$ ) form the CTQ subscales indicated that any experience of childhood trauma was also high: Emotional Abuse $82.1 \%$, Physical Abuse $67.7 \%$, Sexual Abuse 55.9\%, Emotional Neglect 83.1\%, and Physical Neglect 66.7\%.

Positive status rates for each of the six ICD-11 CPTSD Scale dimensions are shown in Table 2. The diagnostic rates were very high for all PTSD dimensions 92.7-97.9\% and lower for the DSO dimensions $68.2-72.5 \%$. The mean scores for each of the PTSD items (range 2.26-3.20) were higher than for the DSO items (range 1.20-2.85).

The fit statistics for the LCA analyses are reported in Table 3.

The 2-class solution produced the lowest values for the BIC and the LRT became non-significant for the 3-class solution. The lowest AIC and ssaBIC was for the 3-class solution, although the difference was small compared to the 2-class solution. The 2-class solution was judged the best model based on the BIC (Nylund et al., 2007) and parsimony. The profile plot for this solution is presented in Fig. 1.

Class 1 was the largest $(\mathrm{N}=146,75.6 \%)$ and was characterised by high probabilities of meeting the diagnostic criteria all of the PTSD and DSO variables. This class was labelled the "CPTSD" class. Class 2

Table 2

Frequencies of Meeting Diagnostic Criteria for Six ICD-11 CPTSD Scale dimensions.

\begin{tabular}{lll}
\hline & Count & (\%) \\
\hline Re-experiencing & 189 & $(97.9)$ \\
Avoidance & 188 & $(97.4)$ \\
Hypervigilance & 179 & $(92.7)$ \\
Affective Dysregulation & 140 & $(72.5)$ \\
Negative Self Concept & 131 & $(68.2)$ \\
Disturbed Relationships & 136 & $(70.5)$ \\
\hline
\end{tabular}


Table 3

Fit Statistics for Diagnostic Variables from ICD-11 CPTSD Scale.

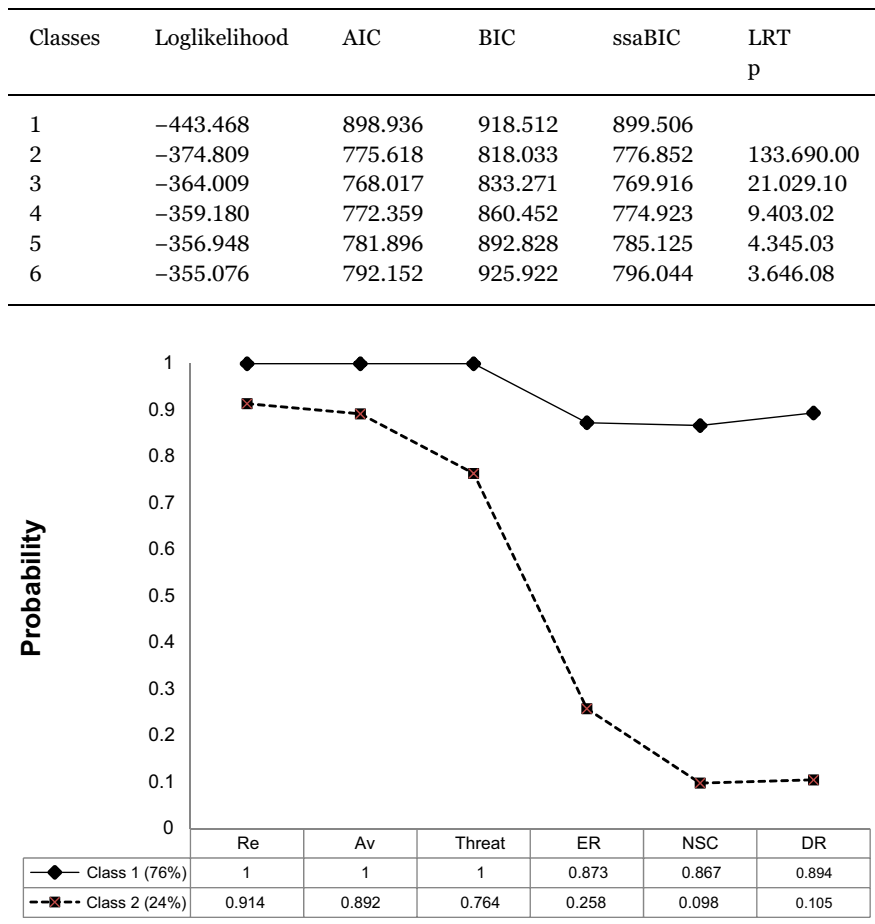

Fig. 1. Profile Plot of Latent Classes of Diagnostic Variables from ICD-TQ Scale. RE=Reexperiencing; $\mathrm{AV}=$ Avoidance; Threat=Sense of threat; $\mathrm{AD}=$ Affective dysregulation; NSC=Negative self-concept; DR=Disturbed relationships.

$(\mathrm{N}=47,24.4 \%)$ had a high probability of meeting the diagnostic criteria for the PTSD variables, but a relatively low probability of meeting the diagnostic criteria for the DSO variables. This class was labelled the "PTSD" class.

There were no significant differences between the classes in terms of gender $\left(\chi^{2}=.300, \mathrm{df}=1, \mathrm{p}=.584\right)$, age $(\mathrm{t}(191)=-1.056, \mathrm{p}=.292)$, or educational attainment $\left(\chi^{2}=3.229, \mathrm{df}=4, \mathrm{p}=.584\right)$. Membership of the CPTSD class was associated with lower likelihood of full-time or parttime employment $\left(\chi^{2}=13.466, \mathrm{df}=5, \mathrm{p}<.05\right)$, lower likelihood of being married $\left(\chi^{2}=17.423, \mathrm{df}=4, \mathrm{p}<.05\right)$, and more likely to be living alone and less likely to be living with a partner or family $\left(\chi^{2}=24.192, \mathrm{df}=3, \mathrm{p}\right.$ $<.05)$. The CPTSD class was also more likely to be receiving psychotropic medication $\left(\chi^{2}=18.383, \mathrm{df}=1, \mathrm{p}<.05\right)$.

In order to test if the CPTSD class would report higher rates of childhood trauma and stressful life events $t$-tests were conducted with class membership as the independent variable and mean scores from the CTQ and summed scores on the LEC as dependent variables. The results are reported in Table 4.

All differences were statistically significant with the CPTSD class

Table 4

Tests of difference between CPTSD and PTSD classes on Trauma Related Measures.

\begin{tabular}{lllllll}
\hline \multirow{2}{*}{ Scale } & \multicolumn{2}{l}{ CPTSD Class } & \multicolumn{2}{l}{ PTSD Class } & & \\
& \cline { 2 - 4 } & Mean (SD) & Mean (SD) & t (df) & $\mathrm{p}$ & $\mathrm{d}$ \\
\hline CTQ: Emotional abuse & $3.06(1.317)$ & $1.95(1.08)$ & $5.24(191)$ & $<.001$ & .88 \\
CTQ: Physical abuse & $2.26(1.21)$ & $1.60(.90)$ & $3.48(191)$ & $<.001$ & .58 \\
CTQ: Sexual abuse & $2.71(1.65)$ & $1.65(1.17)$ & $4.08(191)$ & $<.001$ & .69 \\
CTQ: Emotional & $2.90(1.23)$ & $1.91(1.01)$ & $4.95(191)$ & $<.001$ & .84 \\
$\quad$ & & & & & \\
$\quad$ neglect & & & & & & \\
CTQ: Physical neglect & $2.06(1.04)$ & $1.33(.53)$ & $4.56(191)$ & $<.001$ & .77 \\
Total LEC scores & $5.78(2.50)$ & $4.19(2.55)$ & $3.768(191)$ & $<.001$ & .63
\end{tabular}

Note: $\mathrm{CTQ}=$ Childhood trauma questionnaire; LEC=Life events checklist; $d=$ Cohen's $d$ effect size $(.20=$ small, $.50=$ moderate, $.80=$ large $)$.
Table 5

Childhood Trauma Variables Predicting PTSD and CPTSD Classes.

\begin{tabular}{|c|c|c|c|c|c|}
\hline \multirow[t]{2}{*}{ Trauma } & \multicolumn{2}{|l|}{$\begin{array}{l}\text { CPTSD } \\
\text { Class }\end{array}$} & \multirow[b]{2}{*}{$\chi^{2}(\mathrm{df})$} & \multirow[b]{2}{*}{$\mathrm{p}$} & \multirow[b]{2}{*}{ OR $(95 \% \mathrm{CI})$} \\
\hline & $\mathrm{N}=146$ & $\mathrm{~N}=47$ & & & \\
\hline $\begin{array}{c}\text { Emotional } \\
\text { abuse }\end{array}$ & $\begin{array}{l}127 \\
(87.0 \%)\end{array}$ & 33 (70.2\%) & $7.06(1)$ & $<.01$ & $\begin{array}{l}2.83(1.28- \\
6.24)\end{array}$ \\
\hline Physical abuse & $\begin{array}{l}107 \\
(73.3 \%)\end{array}$ & $25(53.2 \%)$ & $6.64(1)$ & $<.01$ & $\begin{array}{l}2.41(1.22- \\
4.77)\end{array}$ \\
\hline Sexual abuse & $93(63.7 \%)$ & $16(34.0 \%)$ & $12.72(1)$ & $<.001$ & $\begin{array}{l}3.40(1.70- \\
6.78)\end{array}$ \\
\hline $\begin{array}{r}\text { Emotional } \\
\text { neglect }\end{array}$ & $\begin{array}{l}130 \\
(89.0 \%)\end{array}$ & 32 (69.1\%) & $11.58(1)$ & $<.001$ & $\begin{array}{l}3.81(1.70- \\
8.51)\end{array}$ \\
\hline Physical neglect & $\begin{array}{l}109 \\
(74.7 \%)\end{array}$ & 21 (44.7\%) & $14.53(1)$ & $<.001$ & $\begin{array}{l}3.64(1.84- \\
7.24)\end{array}$ \\
\hline
\end{tabular}

Note: The ORs indicate the increase in the likelihood of CPTSD class membership.

having higher mean scores on all the CTQ scales, the CTQ total frequency score and the LEC cumulative index scores. According to the guidelines presented by Cohen (1988) the effects sizes (Cohen's d) are all medium to large with the largest effects for Emotional abuse and Emotional neglect.

In order to determine the relative effects of different, and cumulative, childhood traumas a series of chi-square tests and binary logistic regressions were performed. The childhood trauma variables were the binary variables representing endorsement rates for any item (score > 1) from each CTQ subscale. These variables were also summed to produce a variable, the CTQ cumulative childhood trauma index, with possible scores from 0 to 5 . Table 5 shows that childhood trauma type was significantly associated with class membership, and each childhood trauma increased the likelihood of being in the CPTSD class. The largest effects were for emotional and physical neglect with exposure to these increasing the likelihood of CPTSD class membership by almost 4 times.

Two bivariate logistic regression models were used to test the relationship between (1) childhood cumulative trauma and (2) total cumulative stressful life events. First, the CTQ cumulative index (sum of binary CTQ scores ranging from 0 to 5 ) was used as the independent variable and class membership as the dependent variable in a binary logistic regression. The model was statistically significant $\left(\chi^{2}=25.21\right.$, $\mathrm{df}=1, \mathrm{p}<.001)$ and the effect indicated that each additional trauma type increases the likelihood of membership of the CPTSD class by 1.73 times $(\mathrm{B}=.55, \mathrm{se}=.12, \mathrm{p}<.001 ; \mathrm{OR}(95 \% \mathrm{CI})=1.73 \quad(1.38-2.17))$. Second, the LEC cumulative index (scores ranging from 0 to 16) was used as the independent variable and class membership as the dependent variable in a binary logistic regression. The model was statistically significant $\left(\chi^{2}=14.01, \mathrm{df}=1, \mathrm{p}<.001\right)$ and the effect indicated that each additional stressful event type increases the likelihood of membership of the CPTSD class by 1.30 times $(\mathrm{B}=.26$, $\mathrm{se}=.07$, $\mathrm{p}<.001$; OR $(95 \% \mathrm{CI})=1.30(1.12-1.50)$ ). A third analysis entered both childhood trauma and stressful life events, these scores were positively correlated $(\mathrm{r}=.42, \mathrm{p}<.05)$, as predictors of class membership. The model was statistically significant $\left(\chi^{2}=28.36, \mathrm{df}=2, \mathrm{p}<.001\right)$ and the effect for childhood trauma remained statistically significant with an odds ratio of $1.58(\mathrm{~B}=.46, \mathrm{se}=.12, \mathrm{p}<.001$; OR $(95 \% \mathrm{CI})=1.58$ $(1.24-2.03))$ and the stressful life events was not significant $(B=.14$, $\mathrm{se}=.08, \mathrm{p}>.05$; OR $(95 \% \mathrm{CI})=1.15(.98-1.35))$.

Table 6 shows the differences between the classes on mean scores from the Work and Social Adjustment Scale.

There were significant differences between the classes on all of the WSAS domains. The effects for home management and private leisure activities were medium, but there was a large effect size for social leisure activities and particularly family and relationships.

Overall, results indicate that using the newly developed ICD-TQ two subgroups of treatment-seeking individuals could be empirically dis- 
Table 6

Tests of difference between CPTSD and PTSD classes on Work and Social Adjustment Related Measures.

\begin{tabular}{|c|c|c|c|c|c|}
\hline \multirow[t]{2}{*}{ Scale } & $\begin{array}{l}\text { CPTSD } \\
\text { Class }\end{array}$ & $\begin{array}{l}\text { PTSD } \\
\text { Class }\end{array}$ & & & \\
\hline & Mean (SD) & $\begin{array}{l}\text { Mean } \\
\text { (SD) }\end{array}$ & $t(d f)$ & $\mathrm{p}$ & d \\
\hline $\begin{array}{l}\text { WSAS: Home } \\
\text { management }\end{array}$ & $5.15(2.26)$ & $\begin{array}{l}3.61 \\
(2.50)\end{array}$ & $\begin{array}{l}3.927 \\
(190)\end{array}$ & $<.001$ & .66 \\
\hline $\begin{array}{l}\text { WSAS: Social leisure } \\
\text { activities }\end{array}$ & $6.46(1.71)$ & $\begin{array}{l}4.27 \\
(2.62)\end{array}$ & $\begin{array}{l}6.621 \\
(190)\end{array}$ & $<.001$ & 1.20 \\
\hline $\begin{array}{l}\text { WSAS: Private leisure } \\
\text { activities }\end{array}$ & $5.55(2.18)$ & $\begin{array}{l}3.89 \\
(2.62)\end{array}$ & $\begin{array}{l}4.301 \\
(190)\end{array}$ & $<.001$ & .72 \\
\hline $\begin{array}{l}\text { WSAS: Family and } \\
\text { Relationships }\end{array}$ & $6.32(1.76)$ & $\begin{array}{l}3.55 \\
(2.55)\end{array}$ & $\begin{array}{l}8.313 \\
(189)\end{array}$ & $<.001$ & 1.40 \\
\hline
\end{tabular}

Note: $\mathrm{d}=$ Cohen's $\mathrm{d}$ effect size $(.20=$ small, $.50=$ moderate, $.80=$ large $)$.

tinguished based on different patterns of symptom endorsement; a small group high in PTSD symptoms and a large group high in CPTSD symptoms. CPTSD was strongly associated with childhood traumatic life events and worse functional impairment.

\section{Discussion}

All hypotheses of this study were supported. First, the best LCA solution was for a 2 class solution that represented PTSD and CPTSD as per ICD-11 proposals. Second, the CPTSD class reported greater frequency and greater number of different types of childhood trauma and as well a greater cumulative stressful life events index, although only the effect of childhood trauma remained significant in the multivariate analysis. Third, the CPTSD class reported significantly higher levels of functional impairment across four domains with the largest effects being for family and relationship problems. The study also aimed to examine differences between the PTSD and CPTSD classes on a range of socio-demographic variables. There were no significant differences between the classes in terms of gender, age, or educational attainment but the CPTSD class was associated with lower likelihood of full-time or part-time employment, less likely to be married, and more likely to be living alone and less likely to be living with a partner or family, and more likely to be receiving psychotropic medication.

The fit statistics from the LCA (Table 3 ) indicated that the 2-class solution was the best. The larger class $(76 \%)$ was characterised by very high probabilities of meeting the diagnostic criteria for all PTSD and DSO dimensions and maps clearly onto the ICD-11 specification of CPTSD. The smaller class (24\%) was indicative of a PTSD only class as the probabilities associated with the DSO dimensions were relatively low (all less than .30). These classes are similar to the PTSD and CPTSD classes that were reported previously (Cloitre et al., 2013; Cloitre et al., 2014; Elklit et al., 2014; Knefel et al., 2015). The main difference in this study is that no 'low symptom' class was found. This can be explained in terms of the sample characteristics, as this was a treatment seeking sample rather than a simply trauma-exposed sample.

The findings are adding to the evidence base for the ICD-11 proposals for two distinct conditions following exposure to life events, PTSD and CPTSD. The fact that a significantly higher percentage of participants endorsed a CPTSD diagnosis raises questions about the complexity of traumatic presentations in treatment seeking populations. However, in our sample the majority of participants had reported high rates of childhood traumatic life events, and both childhood and adulthood psychological trauma and childhood traumatisation or multiple traumatisation have been associated with a CPTSD diagnosis in previous research (e.g. Cloitre et al., 2013). The significantly higher functional impairment in the CPTSD class supports the validity of distinguishing between the two disorders particularly as it relates to implications for treatment planning.

The ICD approach to disorders of traumatic stress is dissimilar to the DSM-5 proposal for PTSD which has expanded the diagnosis to include symptoms related to affect dysregulation and negative selfconcept (e.g., Criteria D and E and the specifier or subtype for dissociation), leading to a single disorder with multiple potential symptom profiles types that have little in common. The presentation of two distinct disorders is structurally supported by the taxonomic organization of ICD diagnoses where specifiers and subtypes are rarely used but rather a single conceptual "parent" diagnosis (e.g. Posttraumatic Stress Disorders) is followed by multiple "children" diagnoses (e.g. PTSD and CPTSD). This organization is consistent with the "clinical utility" principle of diagnosis that guides the ICD in so far as evidence suggests that mental health providers disregard subtype/ specifier information (Reed et al. 2011). Moreover, the presence of different risk factors (e.g. childhood trauma), different levels of functional impairment and of course different symptom profiles also contribute to making this distinction meaningful and clinically relevant. It is expected that the selection of treatment interventions and the duration of treatment is likely to differ between the two disorders, given the greater number and diversity of symptoms in CPTSD compared to PTSD, although this remains to be tested.

There were some limitations of this study. First, our findings require replication in larger samples and various trauma exposure populations across different cultures. An important goal will be to streamline the number of symptoms in the DSO clusters to those that reliably distinguish individuals with CPTSD compared to PTSD across time and across cultures. Our sample was fairly homogenous and predominantly consisted of people who had experienced exposure to repeat or numerous types of childhood trauma and the high rates of CPTSD compared to PTSD may be attributable to this fact. As previously mentioned, cumulative childhood trauma is more strongly associated with CPTSD than PTSD (Maercker et al., 2013; Cloitre et al., 2013). Second, in the present study we did not consider diagnostic comorbidities such as depression, anxiety or substance use and in the future it would be useful to explore the nature and type of comorbidities associated with each disorder (e.g. O'Donnell et al., 2004). Preliminary evidence suggests that people meeting the criteria for CPTSD are more impaired by depression, anxiety and sleep disturbances compared to PTSD (Elklit et al., 2014). Third, the LEC includes questions on sexual and physical abuse which may have occurred during childhood and may confound the results when the childhood trauma and stressful life events were examined together. Similarly, there is overlap between the DSO 'Disturbances in Relationships' items and the 'Family and Relationships' item from the Work and Social Adjustment Scale which may account for the large effect size. Fourth, the summed scores of the LEC and the CTQ provide information on the number of different types of trauma exposure, but cannot indicate repeated exposure to the same trauma type. Finally, it is important to mention that the ICD-TQ, as a new scale, still requires further validation with various samples exposed to a variety of traumatic stressors. There are a number of issues that need to be resolved during the validation process including, but not exclusively, identifying the most appropriate (and minimum) number of indicators for each dimension, assessing the best level at which 'symptom endorsement' is determined, deriving optimal cut-off scores for the DSO items, determining the best diagnostic algorithm that combines information from the PTSD and DSO items for classifying 'caseness'.

The high prevalence of CPTSD among the most traumatised highlights the potential benefit of identifying new interventions to aid recovery following this diagnosis. There is substantial evidence suggesting that trauma-focused psychological interventions of 9-12 weeks duration can aid recovery from PTSD (e.g. Foa et al., 2008). It may be preferable to offer a longer course of treatment with different interventions for CPTSD because of the higher number and types of symptoms as well as the more severe functional impairment. To date, 
there are some interventions that have been developed for the treatment of CPTSD which address the three additional symptom clusters including affect dysregulation difficulties, relational and social difficulties, and pervasive negative self-concept (e.g. Cloitre et al., 2011). However, the benefits of different types of interventions of shorter and longer and multi-targeted therapies for CPTSD as compared for PTSD should be subject to future research. If treatment planning which provides different interventions according to the diagnosis of PTSD or CPTSD is found to yield better patient outcomes, the proposed distinction as per ICD 11 proposals may help organize clinical services in an effective and efficient way, particularly with regard to the selection of interventions and the duration of treatment.

Notwithstanding its limitations this is the first study that demonstrated that the newly developed ICD-TQ can adequately distinguish between PTSD and CPTSD. The simple structure with limited symptom features and the conceptual organization of the proposed ICD-11 PTSD and CPTSD promise high clinical utility. Polytraumatisation and level of impairment that are associated with CPTSD may further ease the process of diagnosis and aid treatment management decisions. Development of effective treatments for CPTSD should be a subject for further research.

\section{References}

Andrews, B., 1998. Shame and childhood abuse. In: Gilbert, P., Andrews, B. (Eds.), Shame: Interpersonal Behavior, Psychopathology, and Culture. Oxford University Press, Oxford, 176-190.

Akaike, H., 1987. Factor analysis and the AIC. Psychometrika 52, 317-332.

Badour, C.L., Ojserkis, R., McKay, D., Feldner, M.T., 2014. Disgust as a unique affective predictor of mental contamination following sexual trauma. J. Anxiety Disord. 28, 704-711. http://dx.doi.org/10.1016/j.janxdis.2014.07.007.

Bernstein, D.P., Fink, L., 1998. Childhood Trauma Questionnaire. A Retrospective Selfreport. Manual. San Antonio, TX: The Psycho- logical Corporation. The Psychological Corporation, Harcourt Brace \& Company, San Antonio, TX.

Briere, J., Rickards, S., 2007. Self-awareness, affect regulation, and relatedness: differential sequels of childhood versus adult victimization experiences. J. Nerv. Ment. Dis. 195, 497-503.

Cloitre, M., Courtois, C.C., Charuvastra, A., Carapezza, R., Stolbach, B.C., Green, B.L., 2011. Treatment of complex PTSD: results of the ISTSS expert clinician survey on best practices. J. Trauma Stress 24 (6), 616-627.

Cloitre, M., Garvert, D.W., Brewin, C.R., Bryant, R.A., Maercker, A., 2013. Evidence for proposed ICD-11 PTSD and complex PTSD: a latent profile analysis. Eur. J. Psychotraumatol. 4, 20706.

Cloitre, M., Garvert, D.W., Weiss, B., Carlson, E.B., Bryant, R.A., 2014. Distinguishing PTSD, complex PTSD, and borderline personality disorder: a latent class analysis. Eur. J. Psychotraumatol. 5, 25097. http://dx.doi.org/10.3402/ejpt.v5.25097.

Cloitre, M., Scarvalone, P., Difede, J., 1997. Post-traumatic stress disorder, self and interpersonal dysfunction among sexually revictimized women. J. Trauma Stress, 435-450.

Cohen, J., 1988. Statistical Power Analysis for the Behavioral Sciences 2nd ed.. Lawrence Erlbaum Associates, New Jersey.

Dvir, Y., Ford, J.D., Hill, M., Frazier, J.A., 2014. Childhood maltreatment, emotional dysregulation, and psychiatric comorbidities. Harv. Rev. Psychiatry 22, 149. http:// dx.doi.org/10.1097/HRP.0000000000000014.

Eisinga, R., Grotenhuis, M.T., Pelzer, B., 2012. The reliability of a two-item scale: pearson, Cronbach, or Spearman-Brown?Int. J. Public Health 58, 637-642. http:// dx.doi.org/10.1007/s00038-012-0416-3.

Elklit, A., Hyland, P., Shevlin, M., 2014. Evidence of symptom profiles consistent with posttraumatic stress disorder and complex posttraumatic stress disorder in different trauma samples. Eur. J. Psychotraumatol. 5, 24221. http://dx.doi.org/10.3402/ ejpt.v5.24221.

Foa, E.B., Keane, T.M., Friedman, M.J., Cohen, J., 2008. Effective Treatments for PTSD: Practice Guidelines from the International Society for Traumatic Stress Studies 2nd ed.. The Guilford Press, New York, NY.

Gilbert, P., 2000. The relationship of shame, social anxiety and depression: the role of the evaluation of social rank. Clin. Psychol. Psychother. 7, 174-189. http://dx.doi.org/ 10.1002/1099-0879.

Gray, M.J., Litz, B.T., Wang, J., Lombardo, T.W., 2004. Psychometric properties of the Life Events Checklist. Assessment 11, 330-341.

Harris, H.N., Valentiner, D.P., 2002. World assumptions, sexual assault, depression, and fearful attitudes toward relationships. J. Interpers. Violence 17, 286-305. http:// dx.doi.org/10.1177/0886260502017003004.

Herman, J.L., 1992. Complex PTSD: a syndrome in survivors of prolonged and repeated trauma. J. Trauma Stress 5, 377-391. http://dx.doi.org/10.1002/jts.2490050305.

Jansson-Fröjmark, M., 2014. The work and social adjustment scale as a measure of dysfunction in chronic insomnia: reliability and validity. Behav. Cogn. Psychother. 42, 186-198. http://dx.doi.org/10.1017/S135246581200104X.

Kaltman, S., Krupnick, J., Stockton, P., Hooper, L., Green, B.L., 2005. Psychological impact of types of sexual trauma among college women. J. Trauma Stress 18 (5), 547-555.

Knefel, M., Garvert, D.W., Cloitre, M., Lueger-Schuster, B., 2015. Update to an evaluation of ICD-11 PTSD and complex PTSD criteria in a sample of adult survivors of childhood institutional abuse by Knefel \& Lueger-Schuster (2013): a latent profile analysis. Eur. J. Psychotraumatol. 6, 25290. http://dx.doi.org/10.3402/ ejpt.v6.25290.

Kucharska, J., 2015. Sexual and non-sexual trauma, depression and self-esteem in a sample of Polish women. A cross-sectional study. Clin. Psychol. Psychother.. http:// dx.doi.org/10.1002/cpp.1994.

Lo, Y., Mendell, N., Rubin, D.B., 2001. Testing the number of components in a normal mixture. Biometrika 88, 767-778.

Maercker, A., Brewin, C.R., Bryant, R.A., Cloitre, M., Reed, G.M., Van Ommeren, M., Saxena, S., 2013. Proposals for mental disorders specifically associated with stress in the international classification of diseases. Lancet 381 (9878), 1683-1685.

Morina, N., Ford, J.D., 2008. Complex sequelae of psychological trauma among Kosovar civilian war victims. Int. J. Soc. Psychiatry 54 (5), 425-436.

Mundt, J.C., Marks, I.M., Shear, M.K., Greist, J.H., 2002. The work and social adjustment Scale: a simple measure of impairment in functioning. Br. J. Psychiatry 180, 461-464.

Muthén L.Muthén, B., 2012. Mplus User Guide. Version 7. Los Angeles: Statmodel.

Nylund, K.L., Asparouhov, T., Muthen, B., 2007. Deciding on the number of classes in latent class analysis and growth mixture modeling. A Monte Carlo simulation study. Struct. Equ. Model. 14, 535-569.

O’Donnell, M.L., Creamer, M., Pattison, P., 2004. Posttraumatic stress disorder and depression following trauma: understanding comorbidity. Am. J. Psychiatry 161 (8), 1390-1396.

Perkonigg, A., Hofler, M., Cloitre, M., Wittchen, H.U., Trautmann, S., Maercker, A., 2015. Evidence for two different ICD-11 posttraumatic stress disorder in a community sample of adolescents and young adults. Eur. Arch. Psychiatry Clin. Neurosci.. http://dx.doi.org/10.1007/s00406-015-0639-4.

Reed, G.M., 2010. Toward ICD-11: improving the clinical utility of WHO's international classification of mental disorders. Prof. Psychol.: Res. Pract. 41, 457-464.

Reed, G.M., Correia, J.M., Esparza, P., Saxena, S., Maj, M., 2011. The WPA-WHO global survey of psychiatrists' attitudes towards mental disorders classification. World Psychiatry 10, 118-131.

Roth, S., Newman, E., Pelcovitz, D., van der Kolk, B., Mandel, F.S., 1997. Complex PTSD in victims exposed to sexual and physical abuse: results from the DSM-IV Field Trial for Posttraumatic Stress Disorder. J. Traum. Stress 10, 539-555.

Schwartz, G., 1978. Estimating the dimension of a model. Ann. Stat. 6, 461-464.

Sclove, S.L., 1987. Application of model-selection criteria to some problems in multivariate analysis. Psychometrika 52, 333-343.

Street, A.E., Gibson, L.E., Holohan, D.R., 2005. Impact of childhood traumatic events, trauma-related guilt, and avoidant coping strategies on PTSD symptoms in female survivors of domestic violence. J. Trauma. Stress 18, 245-252, (10.1002/jts.20026).

Wolf, E.J., Miller, M.W., Kilpatrick, D., Resnick, H.S., Badour, C.L., Marx, B.P., et al., 2015. ICD-11 Complex PTSD in US National and Veteran Samples: prevalence and Structural Associations with PTSD. Clin. Psychol. Sci. 3, 215-229.

Yuan, K.H., Bentler, P.M., 2000. Three likelihood-based methods for mean and covariance structure analysis with nonnormal missing data. Sociol. Methodol. 30, 165-200. http://dx.doi.org/10.1111/0081-1750.00078.

Zahra, D., Qureshi, A., Pooler, J., Henley, W., Taylor, R., Byng, R., 2014. The work and social adjustment scale: reliability, sensitivity, and value. Int. J. Psychiatry Clin. Pract. 18 (2), 131-138. 